\title{
Thymic stromal lymphopoietin: Next research hotspot of carcinogenesis?
}

\author{
Tong Wu, Juan Wang, Lihua Jia, Bin Cheng*
}

Department of Oral Medicine, Guanghua School of Stomatology, Sun Yat-sen University, Guangzhou, China

Email: ${ }^{*}$ chengbin@mail.sysu.edu.cn

Received 2 August 2012; revised 8 September 2012; accepted 19 September 2012

\begin{abstract}
Thymic stromal lymphopoietin (TSLP) is an epithetlial cell derived cytokine which has been reported to be a master regulator in $T$ helper (Th) 2 driven inflammation. Through acting on dentritic cells (DCs), granulocytes, natural killer $T$ cells or directly on CD4+ $T$ cells, TSLP plays significant roles in the pathogenesis of atopic diseases consisting of the triad of asthma, allergic rhinitis and atopic dermatitis. Recently mounting evidence demonstrated that cancerrelated inflammation play decisive roles at different stages of tumor development, including initiation, promotion, malignant conversion, invasion, and metastasis. As a crucial regulator of Th2 driven inflammation, the involvement of TSLP in carcinogenesis have attracted researchers' attention. However, the mechanisms of TSLP's involvement in carcinogenesis are still largely unknown. In this review we first outline the roles of TSLP involved in allergic inflammation and then we further focus on the recent findings on TSLP's tumor promoting activities hoping to provide hints on elucidation of the TSLP implication in carcinogenesis in future studies.
\end{abstract}

Keywords: Thymic Stromal Lymphopoietin; Inflammation; Cancer; Carcinogenesis

\section{INTRODUCTION}

Thymic stromal lymphopoietin (TSLP), an epithelially derived cytokine initially identified as a bioactive factor secreted in the supernatants of a murine thymic stromal cell line in 1994 [1]. TSLP is now known to have impacts on hematopoietic and nonhematopoietic cell including B cells, basophils, eosinophils, mast cells, DCs, CD4, CD8, and NK T cells, and epithelial cells. As a master regulator of $\mathrm{T}$ helper (Th) 2 driven inflammation, TSLP is capable of activating dendritic cells to promote $\mathrm{T}$ helper (Th) 2 immune responses and directly promote

"Corresponding author.
Th2 differentiation of naïve CD4+ T cell and Th2 cytokine-associated inflammation. [2-6]. TSLP-induced Th2 responses are associated with the pathogenesis of allergic inflammatory diseases, including atopic dermatitis, asthma, and rhinitis. Recently mounting evidence demonstrated that cancer-related inflammation play decisive roles at different stages of tumor development, including initiation, promotion, malignant conversion, invasion, and metastasis [7-9]. So as a strong mediator in inflamemation, the role of TSLP in cancer development has aroused a great deal of interests [10-12]. However, the mechanisms of TSLP's involvement in carcinogenesis are still largely unknown. In this review we outline the roles of TSLP involved in allergic inflammation and then we further focus on the recent finding on TSLP's tumor promoting activities.

\section{TSLP AND ITS RECEPTOR}

Thymic stromal lymphopoietin (TSLP), an epithelially derived cytokine is predominantly expressed by epithetlial cells in thymus, lung, skin, intestine and tonsils as well as stromal cells and mast cells [1,13]. The mouse TSLP gene is located on chromosome 18, while the human TSLP gene is located on chromosome 5q22.1 next to the atopic cytokine cluster on 5q31 [14]. TSLP originnated from both mouse and human exert their biological activities by binding to a heterodimeric receptor that consists of the IL-7 receptor $\alpha$-chain (IL-7R $\alpha$ ) and the TSLP receptor chain (TSLPR), which has low affinity for TSLP, but in combination with IL-7R $\alpha$ generates a high affinity binding site for TSLP and triggers signaling [15]. TSLP, which is constitutively expressed in human thymus, is responsible for the differentiation of $\mathrm{T}$ regulatory (Treg) cells by modulating the activity of thymic DCs. In contrast to the restricted expression of TSLP, TSLPR is more widely detected on many immune cell types, including dendritic cells (DCs), T cells, B cells, mast cells, natural killer T cells (NKT) and monocytes as well as in tissues from heart, skeletal muscle, kidney and liver which suggests that TSLP can function on a broad 
range of cell types [16]. Although cross-species homology for human and mouse TSLP and its receptor is relatively low, both TSLP-TSLPR interactions activate similar signaling pathways such as the transcription factor signal transducer and activator of transcription 3 (STAT3) in human and STAT5 in mouse and human [1].

\section{TSLP AND ALLERGIC INFLAMMATION}

TSLP has been reported to play a critical role in CD4+ T cell homeostasis in the peripheral mucosa-associated lymphoid tissues and in the positive selection and/or expansion of regulatory $\mathrm{T}$ cells in the thymus under normal physiological conditions [17]. In pathological condition, TSLP plays significant roles both at the induction phase of the Th2 response via polarization of DCs to drive Th2 cell differentiation and at the effector phase of the response by promoting the expansion of activated $\mathrm{T}$ cells and their secretion of Th2 cytokines [1]. This Th2 skewing properties of TSLP are strongly associated with the pathogenesis of atopic diseases consisting of the triad of asthma, allergic rhinitis and atopic dermatitis, which are characterized Th 2 cytokine-dominanted inflammatory.

In skin inflammatory conditions such as atopic dermatitis, TSLP expression in the epidermis of lesional skin is higher than that in uninvolved skin or skin of nonallergic people. Further more the skin-resident DCs in patients with atopic dermatitis have a more activated phenotype, which may migrate toward the draining lymph node to prime CD4+ T cells. TSLP can induce OX40L expression on DCs, after primed by TSLP-activated DCs via OX40-OX40L interaction, CD4 $+\mathrm{T}$ cells differentiate into inflammatory Th2 effecter and memory cells, and thus initiate the adaptive phase of allergic immune responses $[18,19]$. In mouse models, only the increasing TSLP concentrations in the epidermis can induce the onset of Th2 cytokine-associated inflammation, which has all the cardinal features of human atopic dermatitis [20].

TSLP has recently been proved to be a key pro-allergic cytokine in chronic airway diseases, such as asthma and chronic obstructive pulmonary disease (COPD) for the detection of high levels expression of TSLP in bronchial mucosa [21]. The animal experiment demonstrated that TSLP is both necessary and sufficient for the development of Th2 cytokine-associated inflammation of the airways. Mice expressing a TSLP transgene in the airway epithelium develop a spontaneous, progressive inflamematory disease with all the characteristics of human asthma, TSLP was capable of activating bone marrowderived dendritic cells to upregulate costimulatory molecules and produce the $\mathrm{T}$ helper type 2 cell-attracting chemokine CCL17 [22]. On the contrary, TSLP receptor- deficient mice failed to develop asthma in response to inhaled antigen [23].

\section{TSLP AND CARCINOGENESIS}

Recently researches have demonstrated that immune responses have involved and play a vital role in several stages of tumor development, such as initiation, promotion, malignant conversion, invasion, and metastasis [79]. As a crucial regulator of Th2 driven inflammation, the involvement of TSLP in carcinogenesis have attracted researchers' attention. The available researches shown that TSLP is associated with several cancer including lung, pancreatic and breast cancer [10-12]. However, the possible regulatory mechanisms of TSLP underlying these cancers are not clear and varied in different tumor.

\subsection{Cellular Source and Target of TSLP in Tumor Microenvironment}

Similar to normal skin, lung epithelium, breast epithelial cells, lung cancer cell and breast cancer cells have the capacity to express TSLP $[10,12]$. In contrast with breast cancer in which TSLP expression is specific to epithelial cells and no staining can be found in tumor-infiltrating fibroblasts [12]. One recent research on pancreatic cancer demonstrated that cancer-associated fibroblasts (CAFs) secreted TSLP were an important tumor promoter in cancer progression [11]. These experiments shown that TSLP expression is up-regulated in pancreatic cancer and released by CAFs under the influence of TNF- $\alpha$ and IL- $1 \beta$ that are secreted by tumor cells. So in tumor microenvironment, the ability of tumor epithelium and stroma to secrete TSLP varied according to tumor content dependent manner. The cellular target of TSLP include DCs and T cells which induce carcinogenesis through different mechanisms subsequently [10-12,24].

\subsection{TSLP in Lung Cancer}

One of the important mechanisms for cancer to escape immune surveillance is to create an immunosuppressive microenvironment. It has been reported that Tregs, which could suppress the activity of lymphocytes and help the tumor cells to escape the host immune system, were increased in the peripheral blood or tumor microenvironment in patients with cancer [25-28]. In lung cancer, the prevalence of Tregs in tumor microenvironment was correlated with the expression of TSLP which was significantly increased compared with that in benign lesion and non-cancer lung tissue and correlated with pathologic type, stage, tumor size, and lymph node metastasis [10]. Further study demonstrated that TSLP was capable of inducing the differentiation of CD4+ CD25- T cells 
into CD4+ CD25+ Tregs and the subsequent migration of Tregs to the cancer microenvironment by secreting chemokines MDC/CCL22 and TARC/CCL17 in a DCdependent manner [10].

\subsection{TSLP in Pancreatic Cancer}

One recent research on pancreatic cancer demonstrated that TSLP expression is up-regulated in pancreatic cancer and released by CAFs under the influence of TNF- $\alpha$ and IL- $1 \beta$ that are secreted by tumor cells [11]. In vitro study found that myeloid DCs are activated with features of TSLP-treated DCs by the supernatant of proinflammatory cytokine-treated CAFs and acquire Th2-polarizing capability. In vivo study shown that DCs with of TSLP-treated and Th2-attracting features are present in pancreatic cancer patients. Collectively, based on these available results the authors proposed a hypothesis on how CAFs secreted TSLP promote Th2-mediated inflammation in pancreatic cancer which correlated with reduced survival in pancreatic cancer under the influence of tumor cells. Pancreatic tumor cells release proinflammatory cytokines (TNF- $\alpha$ and IL- $1 \beta$ ) and elicit the secretion of TSLP by CAFs. These CAFs-derived TSLP activate tumor antigen-load resident $\mathrm{DC}$ and induce their migration to draining lymph nodes where they activate tumor antigen-specific CD4+ Th2 cells. Finally these CD4+ Th2 cells home to the tumor under the influence of tumor-derived Th2 chemoattractants to exert tumorpromoting effecter functions.

\subsection{TSLP in Breast Cancer}

Besides pancreatic cancer, breast cancer which were infiltrated with tumor promoting inflammatory Th2 cell are also driven by breast cancer-derived TSLP [12]. TSLP secreted by breast cancer cells induced OX40L-expressing DCs migration to the tumor microenvironment which can smolder type 2 inflammation that perpetuates breast cancer. Further more, another research in mice on breast cancer demonstrated that CD4+ T cells are also targets of cancer-produced TSLP during cancer progresssion and metastasis [24]. TSLP was responsible for the lung metastasis by inducing production of CCL17 in the lungs and non-Treg subsets of CD4+ T cells, whereas DCs did not appear to be critical in this process.

\section{PERSPECTIVE}

The evidence available to date indicates that the cancerpromoting activity of TSLP from cancer cell or CAFs varied in different cancers primarily required signaling through the TSLP receptor on DCs or CD4+ T cells, promoting Th2-skewed immune responses and production of immunosuppressive factors. However, many de- tails underlying effect of TSLP on cacinogenesis such as signal transduction mechanisms are largely unknown. Our previous study found that TSLP was expressed on mouse oral keratinocyte under inflammation [29], and TSLP and its receptor were detected on oral squamous cell carcinoma cell line (unpublished data). Whether TSLP is also involved in the carcinogenesis in other epithelial tumor type which progression have been reported to be associated with immune disregulation and elevated Th2 cytokines expression such as head and neck carcinoma remains to be fully elucidated in further study $[30,31]$. Notably, elucidation of the TSLP implication in carcinogesesis may offer novel therapeutic options to complement currently available therapeutic strategies.

\section{ACKNOWLEDGEMENTS}

This work was supported by grants from the National Natural Science Foundation of China (No. 91029712) and the Fundamental Research Funds for the Central Universities of China (2009).

\section{REFERENCES}

[1] He, R. and Geha, R.S. (2010) Thymic stromal lymphopoietin. Annals of the New York Academy of Sciences, 1183, 13-24. doi:10.1111/j.1749-6632.2009.05128.x

[2] Ito, T., Wang, Y.H., Duramad, O., et al. (2005) TSLPactivated dendritic cells induce an inflammatory $\mathrm{T}$ helper type 2 cell response through OX40 ligand. Journal of Experimental Medicine, 202, 1213-1223. doi:10.1084/jem.20051135

[3] Lambrecht, B.N. and Hammad, H. (2009) Biology of lung dendritic cells at the origin of asthma. Immunity, 31, 412-424. doi:10.1016/j.immuni.2009.08.008

[4] Zhang, Y. and Zhou, B. (2012) Functions of thymic stromal lymphopoietin in immunity and disease. Immunology Research, 52, 211-223. doi:10.1007/s12026-012-8264-z

[5] Roan, F., Bell, B.D., Stoklasek, T.A., Kitajima, M., Han, H. and Ziegler, S.F. (2012) The multiple facets of thymic stromal lymphopoietin (TSLP) during allergic inflammation and beyond. Journal of Leukocyte Biology, 91, 877886. doi:10.1189/jlb.1211622

[6] Takai, T. (2012) TSLP expression: Cellular sources, triggers, and regulatory mechanisms. Allergology International, 61, 3-17. doi:10.2332/allergolint.11-RAI-0395

[7] Colotta, F., Allavena, P., Sica, A., et al. (2009) Cancerrelated inflammation, the seventh hallmark of cancer: Links to genetic instability. Carcinogenesis, 30, 10731081. doi:10.1093/carcin/bgp127

[8] Grivennikov, S.I., Greten, F.R. and Karin, M. (2010) Immunity, inflammation, and cancer. Cell, 140, 883-899. doi:10.1016/j.cell.2010.01.025

[9] Hanahan, D. and Weinberg, R.A. (2011) Hallmarks of cancer: The next generation. Cell, 144, 646-674. doi:10.1016/j.cell.2011.02.013

[10] Li, H., Zhao, H., Yu, J., Su, Y., Cao, S., An, X. and Ren, 
$X$. (2011) Increased prevalence of regulatory $T$ cells in the lung cancer microenvironment: A role of thymic stromal lymphopoietin. Cancer Immunology, Immunotherapy, 60, 1587-1596. doi:10.1007/s00262-011-1059-6

[11] De Monte, L., Reni, M., Tassi, E., et al. (2011) Intratumor $\mathrm{T}$ helper type 2 cell infiltrate correlates with cancer-associated fibroblast thymic stromal lymphopoietin production and reduced survival in pancreatic cancer. Journal of Experimental Medicine, 208, 469-478. doi:10.1084/jem.20101876

[12] Pedroza-Gonzalez, A., Xu, K., Wu, T.C., et al. (2011) Thymic stromal lymphopoietin fosters human breast tumor growth by promoting type 2 inflammation. Journal of Experimental Medicine, 208, 479-490. doi:10.1084/jem.20102131

[13] Friend, S.L., Hosier, S., Nelson, A., et al. (1994) A thymic stromal cell line supports in vitro development of surface IgM+ B cells and produces a novel growth factor affecting $\mathrm{B}$ and $\mathrm{T}$ lineage cells. Experimental Hematology, 22, 321-328.

[14] Quentmeier, H., Drexler, H.G., Fleckenstein, D., et al. (2001) Cloning of human thymic stromal lymphopoietin (TSLP) and signaling mechanisms leading to proliferation. Leukemia, 15, 1286-1292. doi:10.1038/sj.leu.2402175

[15] Pandey, A., Ozaki, K., Baumann, H., et al. (2000) Cloning of a receptor subunit required for signaling by thymic stromal lymphopoietin. Nature Immunology, 1, 59-64.

[16] Ziegler, S.F. and Artis, D. (2010) Sensing the outside world: TSLP regulates barrier immunity. Nature Immunology, 11, 289-293. doi:10.1038/ni.1852

[17] Watanabe, N., Wang, Y.H,, Lee, H.K., et al. (2005) Hassall's corpuscles instruct dendritic cells to induce CD4+ $\mathrm{CD} 25+$ regulatory $\mathrm{T}$ cells in human thymus. Nature, $\mathbf{4 3 6}$, 1181-1185. doi:10.1038/nature03886

[18] Wang, Y.H. and Liu, Y.J. (2007) OX40-OX40L interactions: A promising therapeutic target for allergic diseases? The Journal of Clinical Investigation, 117, 3655-3657. doi:10.1172/JCI34182

[19] Liu, Y.J. (2007) Thymic stromal lymphopoietin and OX40 ligand pathway in the initiation of dendritic cellmediated allergic inflammation. Journal of Allergy and Clinical Immunology, 120, 238-244. doi:10.1016/j.jaci.2007.06.004

[20] Yoo, J., Omori, M., Gyarmati, D., et al. (2005) Spontaneous atopic dermatitis in mice expressing an inducible thymic stromal lymphopoietin transgene specifically in the skin. Journal of Experimental Medicine, 202, 541-549. doi:10.1084/jem.20041503

[21] Redhu, N.S. and Gounni, A.S. (2011) Function and mechanisms of TSLP/TSLPR complex in asthma and COPD. Clinical \& Experimental Allergy, 42, 994-1005. doi:10.1111/j.1365-2222.2011.03919.x

[22] Zhou, B., Comeau, M.R., De Smedt, T., et al. (2005) Thymic stromal lymphopoietin as a key initiator of allergic airway inflammation in mice. Nature Immunology, $\mathbf{6}$, 1047-1053. doi: $10.1038 /$ ni1247

[23] Al-Shami, A., Spolski, R., Kelly, J., et al. (2005) A role for TSLP in the development of inflammation in an asthma model. Journal of Experimental Medicine, 202, 829-839. doi:10.1084/jem.20050199

[24] Olkhanud, P.B., Rochman, Y., Bodogai, M., et al. (2011) Thymic stromal lymphopoietin is a key mediator of breast cancer progression. Journal of Immunology, 186, 56565662. doi:10.4049/jimmunol.1100463

[25] Ohara, M., Yamaguchi, Y., Matsuura, K., et al. (2009) Possible involvement of regulatory $\mathrm{T}$ cells in tumor onset and progression in primary breast cancer. Cancer Immunology, Immunotherapy, 58, 441-447. doi:10.1007/s00262-008-0570-x

[26] Li, L., Chao, Q.G., Ping, L.Z., et al. (2009) The prevalence of FOXP3+ regulatory T-cells in peripheral blood of patients with NSCLC. Cancer Biotherapy and Radiopharmaceuticals, 24, 357-367. doi:10.1089/cbr.2008.0612

[27] Tokuno, K., Hazama, S., Yoshino, S., et al. (2009) Increased prevalence of regulatory T-cells in the peripheral blood of patients with gastrointestinal cancer. Anticancer Research, 29, 1527-1532.

[28] Strauss, L., Bergmann, C., Szczepanski, M., et al. (2007) A unique subset of CD4+CD25highFoxp3+ $\mathrm{T}$ cells secreting interleukin-10 and transforming growth factorbetal mediates suppression in the tumor microenvironment. Clinical Cancer Research, 13, 4345-4354. doi:10.1158/1078-0432.CCR-07-0472

[29] Wu, T., Jia, L., Du, R., et al. (2011) Genome-wide analysis reveals the active roles of keratinocytes in oral mucosal adaptive immune response. Experimental Biology and Medicine, 236, 832-843. doi:10.1258/ebm.2011.010307

[30] Bose, A., Chakraborty, T., Chakraborty, K., et al. (2008) Dysregulation in immune functions is reflected in tumor cell cytotoxicity by peripheral blood mononuclear cells from head and neck squamous cell carcinoma patients. Cancer Immunity, 8, 10.

[31] Agarwal, A., Rani, M., Saha, G.K., et al. (2003) Disregulated expression of the Th2 cytokine gene in patients with intraoral squamous cell carcinoma. Immunological Investigations, 32, 17-30. doi:10.1081/IMM-120019205

Treg: T Regulatory Cell

NKT: Natural Killer T Cells

STAT: Signal Transducer and Activator of Transcription

COPD: Chronic Obstructive Pulmonary Disease

CAF: Cancer-Associated Fibroblast 